\title{
Theoretical status of single top and top associated production predictions
}

\section{Rikkert Frederix*}

Physik Department T31, Technische Universität München, James-Franck-Str. 1, D-85748 Garching, Germany

E-mail: rikkert.frederixetum.de

In this talk the recent progress in theoretical developments for single top production and top pair production in association with heavy vector bosons is reviewed. For top pair associated production, particular emphasis is put on complete-NLO predecitions, i.e. predictions including all LO and NLO contributions in an expansion in both the strong and the electroweak couplings. For single-top production the three most relevant results that appeared this year are summarized.

Sixth Annual Conference on Large Hadron Collider Physics (LHCP2018)

4-9 June 2018

Bologna, Italy

${ }^{*}$ Speaker. 


\section{Introduction}

In the quest for understanding the Standard Model (SM) of particle physics, and possibly finding the physics beyond it, careful comparisons between experimental data and theoretical predictions need to be made. In particular, with the constantly growing, highly accurate data sample at the LHC, more and more SM process can be measured with high accuracy. Most exciting in this context is the measurement of Higgs boson production in association with the top quark pair, announced at this conference [1,2]. This confirms that the Higgs couples strongly to the top quark and is most-likely (at least to a certain extend) responsible for the generation of its mass. Cross sections of similar size have been measured for the production of a top quark pair in association with a heavy vector boson $[3,4]$. Indeed, these measurements can be used to measure the top quark coupling to the Higgs and $Z$ boson.

The third heavy boson in the SM, the $W^{ \pm}$boson, couples also to the top quark. To measure its coupling one has to look at single top quark production, because a quark changes flavour when exchanging a $W^{ \pm}$boson, and therefore is typically not produced in pairs. The single top quark production was first measured at the Tevatron [5, 6], but recently the first differential distributions have appeared at the LHC [7, 8].

In order to make definite conclusions from the measurements of these processes, they need to be interpreted within a theory model. In particular, if we could find an inconsistency between the SM and these measurements we would have a clear signal for the need of New Physics. To get the most out of the measurements in this context, highly accurate predictions for these processes within the SM are required. In this talk, the latest developments in the predictions for the top associated production processes as well as the single-top production mechanisms are presented.

\section{Top associated production with a heavy vector boson}

Even at next-to-leading order (NLO) in the strong coupling the total cross section for production of top-anti-top quark pair together with a heavy boson has a relatively large perturbative uncertainty from renormalisation and factorisation scale dependence - of the order of 10-15\% at the $13 \mathrm{TeV}$ LHC. It would therefore be interesting to compute also the next order (NNLO) in the strong coupling to reduce this uncertainty. However, since this has already been a formidable task for top pair production (without the extra, heavy boson) [9], the complete NNLO calculation for $t \bar{t} H, t \bar{t} Z$ and $t \bar{t} W^{ \pm}$production are completely out of reach with current technology.

On the other hand, certain subsets of the higher order corrections are known at all orders in perturbation theory. In particular, since these processes feature relatively heavy particles in the final state, with a total invariant mass typically $600-700 \mathrm{GeV}$, it can be expected that effects due to being forced to be relatively close to that invariant mass threshold, because there is not much energy left in the collision to produce hard radiation in association with the core process, is a dominant contribution to higher order corrections. Indeed, recently these so-called threshold logarithms have been resummed to all orders in perturbation theory up to next-to-next-to-leading logarithmic (NNLL) accuracy, including their consistent matching to the full NLO results $[10,11$, $12,13]$. Including these all-order terms reduces the scale dependence by $30-50 \%$, as can be seen in table 1. 


\begin{tabular}{|c|c|c|c|}
\hline pert. order & process & PDF order & $\sigma[\mathrm{fb}]$ \\
\hline NLO & $t \bar{t} H$ & NLO & $474.8_{-51.9}^{+47.2}$ \\
\hline NLO+NNLL & $t \bar{t} H$ & NNLO & $486.4_{-24.5}^{+29.9}$ \\
\hline NLO & $t \bar{t} W^{+}$ & NLO & $356.3_{-39.5}^{+43.7}$ \\
\hline NLO+NNLL & $t \bar{t} W^{+}$ & NNLO & $341.0_{-13.6}^{+23.1}$ \\
\hline NLO & $t \bar{t} W^{-}$ & NLO & $182.2_{-20.4}^{+23.1}$ \\
\hline NLO+NNLL & $t \bar{t} W^{-}$ & NNLO & $177.1_{-6.9}^{+12.0}$ \\
\hline NLO & $t \bar{t} Z$ & NLO & $728.3_{-90.3}^{+93.8}$ \\
\hline NLO+NNLL & $t \bar{t} Z$ & NNLO & $777.8_{-65.2}^{+61.3}$ \\
\hline
\end{tabular}

Table 1: Total cross sections in fb for the LHC at $13 \mathrm{TeV}$. Table taken from ref. [14]

With the uncertainties well below $10 \%$, one might wonder if electroweak (EW) corrections play a significant role in these processes. At the level of total rates, it can be expected that the $\mathrm{NLO}_{1}$ (the NLO QCD corrections), are the largest followed by $\mathrm{NLO}_{2}$ (the NLO EW corrections), $\mathrm{NLO}_{3}$ and finally $\mathrm{NLO}_{4}$ - see fig. 1 for details on the naming convention for the corrections. On the other hand, in tails of distributions, where EW Sudakov logarithms can become sizeable, the $\mathrm{NLO}_{2}$ term typically dominates over the $\mathrm{NLO}_{1}$ corrections.

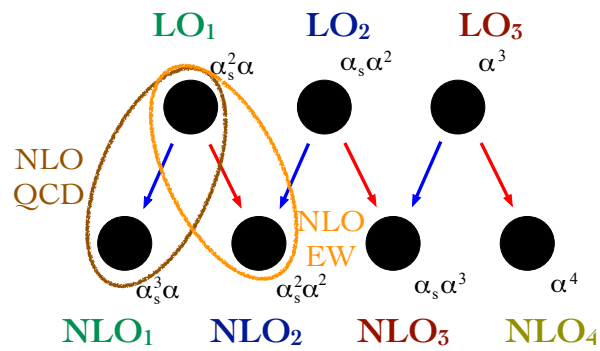

Figure 1: Naming conventions for the various contributions with fixed $\alpha_{S}$ and $\alpha$ coupling combinations to $t \bar{t}+Z / W^{ \pm} / H$. Including all these contributions is referred to as "complete-NLO" predictions.

At the level of total rates the above reasoning on the size of the corrections holds pretty much for the $t \bar{t} Z$ and $t \bar{t} H$ processes, see tab. 2. Also at the level of differential distributions, the size of the contributions to the complete-NLO computation behave as expected for these two processes. See for example the left hand plot of fig. 2 for the various LO and NLO contributions to the transverse momentum of the Higgs boson in $p p \rightarrow t \bar{t} H$ production. ${ }^{1}$ On the other hand, for the $t \bar{t} W^{ \pm}$ processes, there is a surprise: the $\mathrm{NLO}_{3}$ terms is more than an order of magnitude larger than one would expected from the sizes of the EW and QCD coupling strengths, see tab. 2 and the right hand plot of fig. 2. The origin of these large corrections can be pointed to the opening of $t W \rightarrow t W$ scattering in the $\mathrm{NLO}_{3}$ contributions [16]. This is not the only process for which it is known that the subleading $\mathrm{NLO}_{i}$ terms are larger than expected [16, 17]. In particular for 4-top production, the $\mathrm{NLO}_{2}$ and $\mathrm{NLO}_{3}$ contributions are individually quite large, easily surpassing 10-15\% (depending on the scales used), but come with opposite signs. However, when taken together their sum reduces

\footnotetext{
${ }^{1}$ Very recently, in ref. [15] the NLO QCD and EW corrections to this process, including the top quark decays and off-shell effects have been considered.
} 


\begin{tabular}{lccc}
\hline & $p p \rightarrow t \bar{t} Z$ & $p p \rightarrow t \bar{t} W^{+}$ & $p p \rightarrow t \bar{t} H$ \\
\hline $\mathrm{LO}_{1}$ & $5.0463 \pm 0.0003 \cdot 10^{-1} \mathrm{pb}$ & $2.4116 \pm 0.0001 \cdot 10^{-1} \mathrm{pb}$ & $3.4483 \pm 0.0003 \cdot 10^{-1} \mathrm{pb}$ \\
$\mathrm{LO}_{2}$ & $-0.691 \pm 0.001 \%$ & $+0.000 \pm 0.000 \%$ & $+0.406 \pm 0.001 \%$ \\
$\mathrm{LO}_{3}$ & $+2.259 \pm 0.001 \%$ & $+0.962 \pm 0.000 \%$ & $+0.702 \pm 0.001 \%$ \\
$\mathrm{NLO}_{1}$ & $+44.809 \pm 0.028 \%$ & $+49.504 \pm 0.015 \%$ & $+28.847 \pm 0.020 \%$ \\
$\mathrm{NLO}_{2}$ & $-0.846 \pm 0.004 \%$ & $-4.541 \pm 0.003 \%$ & $+1.794 \pm 0.005 \%$ \\
$\mathrm{NLO}_{3}$ & $+0.845 \pm 0.003 \%$ & $+12.242 \pm 0.014 \%$ & $+0.483 \pm 0.008 \%$ \\
$\mathrm{NLO}_{4}$ & $-0.082 \pm 0.000 \%$ & $+0.017 \pm 0.003 \%$ & $+0.044 \pm 0.000 \%$ \\
\hline
\end{tabular}

Table 2: Cross sections for the five $t \bar{t}+X$ processes. The uncertainties quoted are of statistical nature only, originating from the Monte Carlo integration over the phase space. The subleading LO and NLO contributions are given as percentage fractions of $\mathrm{LO}_{1}$. Table adapted from ref. [18].

to a corrections of around $1 \%$ and is almost independent from the scale choice. The origin of this cancelation is currently not understood.
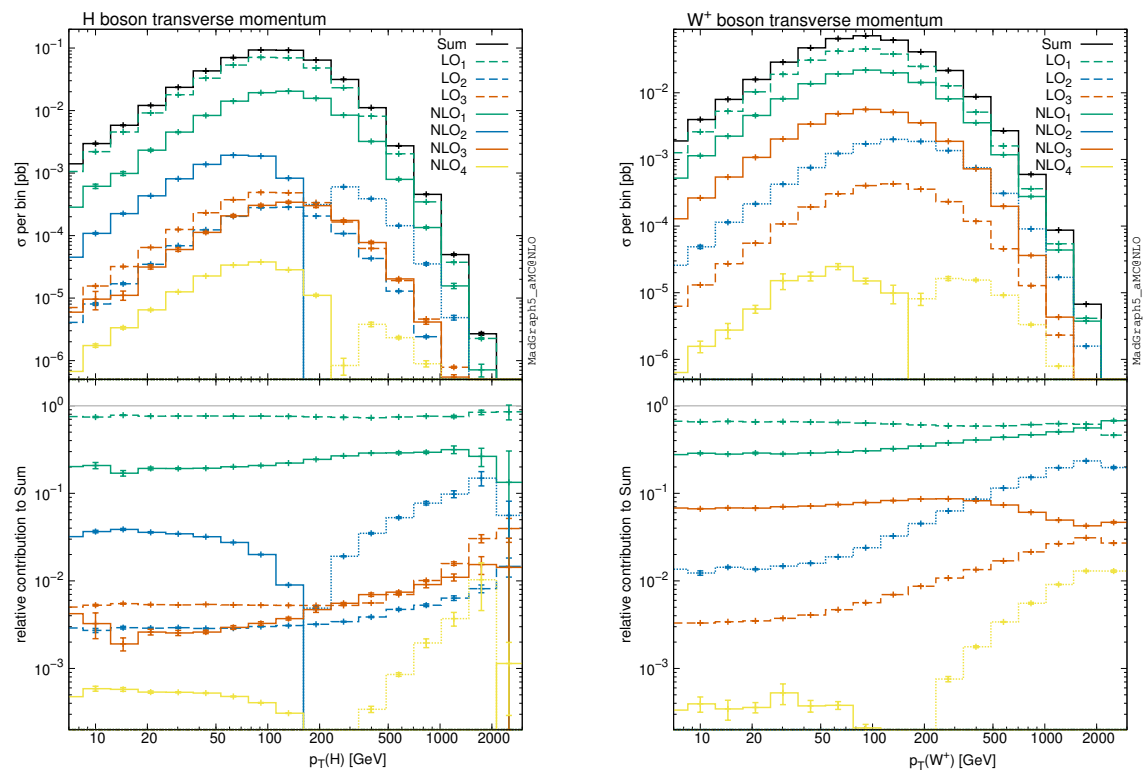

Figure 2: Transverse momenta of the heavy vector boson in $t \bar{t} H$ (left) and $t \bar{t} W^{+}$(right) production. Dashed and solid lines denote positive LO and NLO contributions, respectively, while dot-dashed and dotted are the (absolute value of) negative LO and NLO contributions, respectively. Plots taken from ref. [18].

\section{3. $t$-channel single top prodution}

The three recent developments in single-top production are all related to the largest single top channel, i.e. the production of a single top through a $t$-channel $W$-boson exchange.

\subsection{Transverse momentum resummation}

Earlier this year, predictions in which the transverse momentum logarithms $\log \left(q_{\perp} / Q\right)$, with $Q$ the typical hard scale of the single top process and $q_{\perp}=p_{T}($ top + jet $)$, have been resummed to all 
orders in perturbation theory up to next-to-leading logarithm (NLL) [19]. These predictions should be superior to other known predictions in the region of phase-space where the combined transverse momenta of the top quark and the hardest jet, $q_{\perp}$ is small. These predictions are compared to predictions obtained with the pythia8 [20] parton shower. For central jets agreement is found in the shape of the $q_{\perp}$ distributions. On the other hand, for very forward jets, the resummed predictions peak at much smaller transverse momenta than the pythia8 predictions, see fig. 3. However, in the pythia8 predictions, the recent improvements regarding initial-final dipoles [21] have not been included. It would therefore be interesting to redo this comparison with the updated pythia8 version.

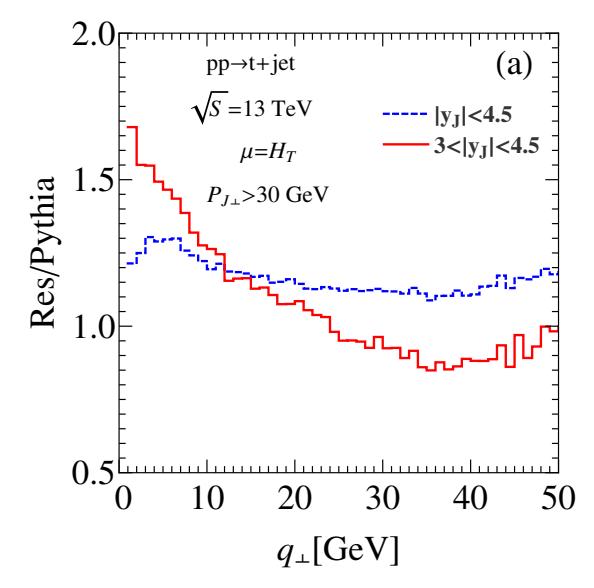

Figure 3: Ratio between the NLL resummed result and the pythia8 predictions for the $q_{\perp}$ distribution. Plot taken from ref. [19].

\subsection{NNLO corrections to production and decay}

In 2017 Berger et al. published the first NNLO calculation for $t$-channel single top production where the corrections are included in both the production and the decay [22]. In this calculation interference between the light and heavy quark linee, the interference between production and decay and any off-shell effects (for the top quark) are neglected. Earlier this year, they updated their results by including more differential distributions and performing a more complete phenomenological study [23]. One of the most interesting results are that the NNLO corrections can be very large and outside the NLO scale uncertainty band in the case where a jet veto is applied, reducing the LO cross section by almost $40 \%$. This is very relevant due to the fact that such a jet veto is applied in experimental analyses in order to reduce the backgrounds from top pair production [8, 24]. Even though such a jet veto might introduce large logarithms order by order in perturbation theory, there are no signs of a breakdown of perturbation theory in the predictions, e.g. through enhanced scale uncertainty bands at higher orders. On the other hand, it would be interesting to see if such a large reduction in rates due to higher orders is well-modeled by a parton shower.

\subsection{Minlo' predictions}

The Minlo' method $[25,26,27]$ is a way of getting predictions that are simultaneously NLO 
accurate for observables differential in a system $S$ and $S+$ jet. In a recent work, this has been applied to $t$-channel single top production resulting in predictions, that are matched to a parton shower, that have NLO accuracy in the $t, t+$ jet and $t+2$ jet phase-spaces [28]. The numerical Minlo' method used for this process, works by enhancing the NLO+PS predictions for the $p p \rightarrow t j j$ process by a NLL $\sigma$ accurate Sudakov form factor, in which an additional term is fitted numerically in such a way that observables inclusive over the 2 nd jet are equal to the NLO+PS predictions for $p p \rightarrow t j$. In fig. 4 we show the transverse momentum of the top quark (left) and the jet multiplicity (right). For the transverse momentum of the top quark, the Minlo' predictions (denoted by STJ ${ }^{\star}$ in the figure) agree with the NLO predictions for $p p \rightarrow t j$ (denoted by ST). Similarly for the 0 and 1jet multiplicities. On the other hand, for the 2-jet rate (and beyond) the Minlo' predictions are in agreement with the NLO predictions for the $p p \rightarrow t j j$ process (denoted by STJ in the figure). This method allows for the inclusion of the NNLO corrections, resulting in the current state-of-the-art for fully exclusive predictions: NNLO+PS.
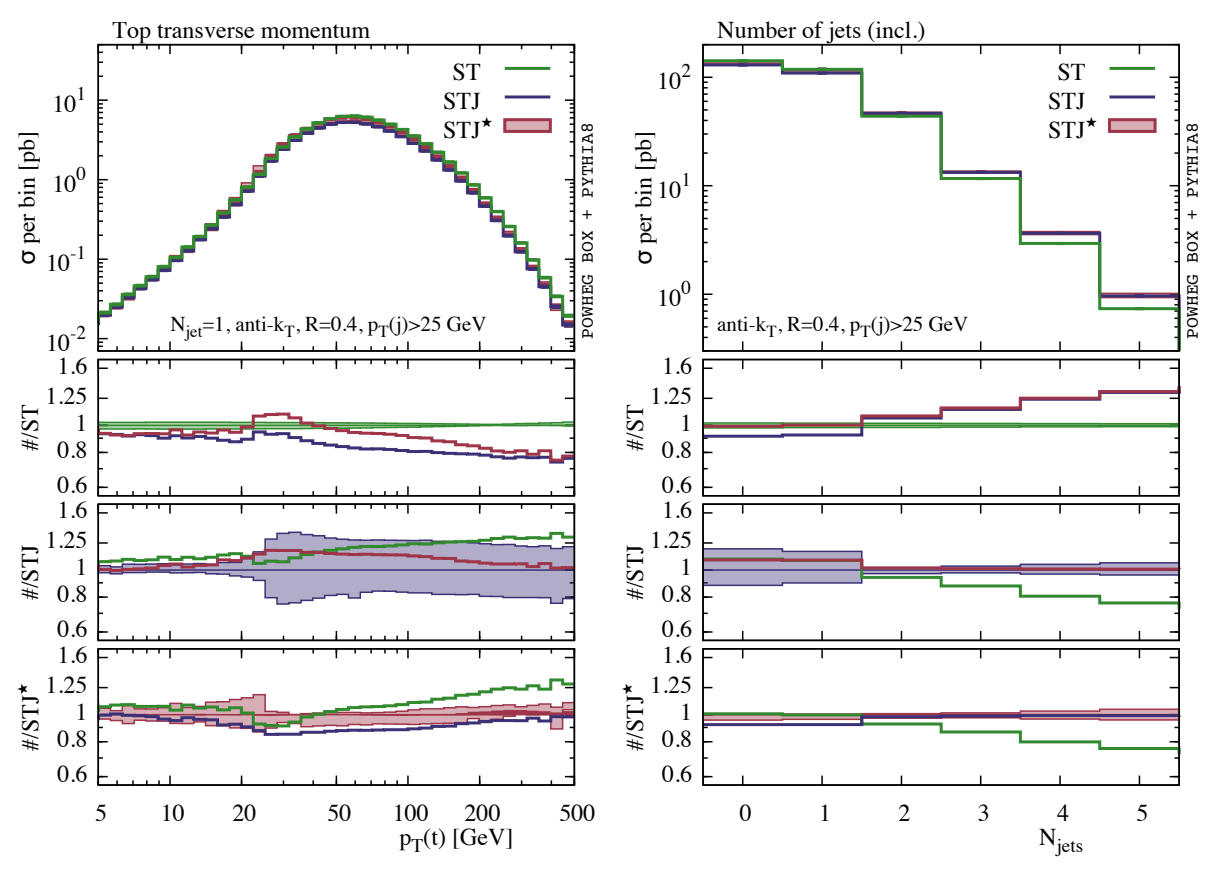

Figure 4: Minlo' predictions for the transverse momentum of the top quark (left) and the jet multiplicity (right). Plots taken from ref. [28].

\section{Conclusions}

In this talk we have summarised the recent developments in top quark pair associated and single top production. For the top quark associated production processes we discussed the matched predictions in which the NLO corrections have been augmented with the resummation of threshold logarithms up to NNLL accuracy. We also showed that the complete-NLO predictions entail no suprises for $t \bar{t} Z$ or $t \bar{t} H$ production. On the other hand, for $t \bar{t} W^{ \pm}$the $\mathrm{NLO}_{3}$ term is much larger than expected, due to the opening of the $t W \rightarrow t W$ scattering contributions. 
For single top production, we mentioned the three latest developments: tranverse momentum resummation to get highly accurate predictions for the $t+$ jet small transverse momentum; a more elaborate study on the NNLO predictions for single-top including, the top decay; and the Minlo' predictions for a NLO accurate predictions in both the single top process, as well as this process plus an additional resolved hard jet, without the inclusion of a merging scale.

\section{Acknowledgements}

The speaker is supported by the Alexander von Humboldt Foundation, in the framework of the Sofja Kovalevskaja Award Project "Event Simulation for the Large Hadron Collider at High Precision".

\section{References}

[1] A. M. Sirunyan et al. [CMS Collaboration], Phys. Rev. Lett. 120 (2018) no.23, 231801.

[2] M. Aaboud et al. [ATLAS Collaboration], Phys. Lett. B 784 (2018) 173.

[3] M. Aaboud et al. [ATLAS Collaboration], Eur. Phys. J. C 77 (2017) no.1, 40.

[4] A. M. Sirunyan et al. [CMS Collaboration], JHEP 1808 (2018) 011.

[5] T. Aaltonen et al. [CDF Collaboration], Phys. Rev. Lett. 103 (2009) 092002.

[6] V. M. Abazov et al. [D0 Collaboration], Phys. Rev. Lett. 103 (2009) 092001.

[7] M. Aaboud et al. [ATLAS Collaboration], Eur. Phys. J. C 78 (2018) no.3, 186.

[8] M. Aaboud et al. [ATLAS Collaboration], Eur. Phys. J. C 77 (2017) no.8, 531.

[9] M. Czakon, P. Fiedler and A. Mitov, Phys. Rev. Lett. 110 (2013) 252004.

[10] A. Broggio, A. Ferroglia, B. D. Pecjak and L. L. Yang, JHEP 1702 (2017) 126.

[11] A. Kulesza, L. Motyka, T. Stebel and V. Theeuwes, Phys. Rev. D 97 (2018) no.11, 114007.

[12] A. Broggio, A. Ferroglia, G. Ossola and B. D. Pecjak, JHEP 1609 (2016) 089.

[13] A. Broggio, A. Ferroglia, G. Ossola, B. D. Pecjak and R. D. Sameshima, JHEP 1704 (2017) 105.

[14] A. Broggio, PoS RADCOR 2017 (2018) 056.

[15] A. Denner, J. N. Lang, M. Pellen and S. Uccirati, JHEP 1702 (2017) 053.

[16] R. Frederix, D. Pagani and M. Zaro, JHEP 1802 (2018) 031.

[17] B. Biedermann, A. Denner and M. Pellen, Phys. Rev. Lett. 118 (2017) no.26, 261801.

[18] R. Frederix, S. Frixione, V. Hirschi, D. Pagani, H.-S. Shao and M. Zaro, JHEP 1807 (2018) 185.

[19] Q. H. Cao, P. Sun, B. Yan, C.-P. Yuan and F. Yuan, arXiv:1801.09656 [hep-ph].

[20] T. Sjöstrand et al., Comput. Phys. Commun. 191 (2015) 159.

[21] B. Cabouat and T. Sjöstrand, Eur. Phys. J. C 78 (2018) no.3, 226.

[22] E. L. Berger, J. Gao, C.-P. Yuan and H. X. Zhu, Phys. Rev. D 94 (2016) no.7, 071501.

[23] E. L. Berger, J. Gao and H. X. Zhu, JHEP 1711 (2017) 158.

[24] A. M. Sirunyan et al. [CMS Collaboration], Phys. Lett. B 772 (2017) 752.

[25] K. Hamilton, P. Nason and G. Zanderighi, JHEP 1210 (2012) 155.

[26] K. Hamilton, P. Nason, C. Oleari and G. Zanderighi, JHEP 1305 (2013) 082.

[27] R. Frederix and K. Hamilton, JHEP 1605 (2016) 042.

[28] S. Carrazza, R. Frederix, K. Hamilton and G. Zanderighi, arXiv:1805.09855 [hep-ph]. 